\title{
Tissue Engineering
}

\section{Daniel J. Gould*}

Baylor College of Medicine, Medical Scientist Training Program, One Baylor Plaza, Houston Texas 77030, USA

\begin{abstract}
Tissue engineering as a field is rapidly developing in order to provide new scaffolds, tissues and organs or devices to replace or supplement function. One key limitation within tissue engineering is the need for rapid perfusion and microvascularization of implanted tissues. Many investigators are currently focused on the rational design of angiogenic tissue engineering scaffolds which can induce the formation of a microvascular host response. This contribution is critical to the field because recently developed tissue engineering products for use in humans lack microvascularization. These successfully engineered thin tissue components include cartilage, bladder and cornea. The lack of microvascularization limits the types of tissue replacements that can be engineered. In order to better understand the need for microvascularization, a basic discussion on the demand for tissue engineering, an overview of current strategies employed by tissue engineering and some successes and challenges within the field must first be presented.
\end{abstract}

\section{Motivation: The Increasing Need for and Cost of Transplantation Fuels Demand for Tissue Engineering}

Many disease processes in the United States involve the destruction or damage of organs and tissues. The cause of destruction of these tissues can originate from ischemic insult triggered by cardiovascular disease, infection, auto-immune attack, cancerous invasion, genetic conditions or toxic exposure. Hundreds of conditions can contribute to the destruction of host organs and ultimately necessitate either assistance of organ function or complete organ transplantation in the host. The most common diseases requiring organ transplant include Polycystic Kidney Disease, Diabetes Mellitus, Chronic Obstructive Pulmonary Disease, Idiopathic Pulmonary Fibrosis, Hypertension, Coronary Heart Disease, Cardiomyopathy, Short Gut Syndrome, Cirrhosis and Hepatitis [1]. In the US, there are currently over 100,000 people awaiting organ transplantation. Those awaiting organ transplantation mostly need kidney transplantation or liver transplantation in fact; these represent approximately $90 \%$ of the people on organ waiting lists. Both liver and kidney tissues are extremely well vascularized, with complex microvascular networks to allow for the diffusion and excretion of waste products and the reabsorption of nutrients and metabolites. There are countless other conditions involving many tissues for which there are no current transplantable methods to alleviate their disease, or to allow recovery of function.

One important consideration of transplantation is the cost associated with this dramatic surgical procedure and the maintenance of the graft for many years following surgery. Of those awaiting transplantation, around 20\% will receive their organ per year, and the cost for organ transplantation in the first year alone will range between $\$ 275,000$ for a pancreas and $\$ 1.1$ million for a heart-lung or intestine transplant [1]. Roughly $\$ 200,000$ of the total cost for organ transplant comes from procurement and immunosuppressants in the first year, however, over time immunosuppression can contribute an additional $\$ 200,000$ to the total cost of transplantation [1]. Furthermore, the process of immunosuppression, though vital to the success of the transplant, can leave the host at risk for numerous infections and the drugs themselves often cause unwanted side effects.

One proposed alternative to tissue transplantation is the development of new tissues and organs derived from cells extracted from the host using technology developed in the field of tissue engineering. The argument for this technology is that it will decrease monetary and human costs by eliminating post transplant immunosuppression and the transportation and surgical costs for procurement of donor organs. Also tissue engineering could provide new options for previously untreatable diseases.

Tissue engineering is a relatively new field of research, which may have roots in ancient practices but was best presented in the landmark paper of Langer and Vacanti in 1993 [2]. Tissue engineering involves the use of three critical ingredients, cells, scaffolding, and cytokines to fabricate tissues as a therapeutic [3,4]. The goal of this field is to replace damaged tissues and organs, in order to provide a greater pool of resources for those awaiting transplantation, as well as to reduce the costs of organ replacement, by reducing immunosuppression required (using host cells) and reducing procurement costs. Furthermore, tissue engineering serves to provide new therapeutic solutions for conditions of tissue loss where transplantation is not currently possible. In many disease conditions this new field will help to better the available treatments by contributing vascularized tissue constructs for implantation; this is particularly relevant to many diseases involving a compromised vascular system.

\section{Limitations of Tissue Engineering: Current Approaches}

Tissue engineering has yet to address the issue of solid, thick tissues, requiring nutrient delivery over more than 150 microns, which is the transport limit of diffusion for most metabolic substrates $[5,6]$. The focus of many labs is to develop microvascularized tissue engineered constructs, in order to provide a solution to the diffusion problem, and to ultimately design and develop thick tissues or whole organs to replace damaged tissues [7]. This represents a major hurdle to tissue engineering and the fabrication of a microvascularized or rapidly perfused tissue construct is a highly sought after goal [5]. One strategy towards this goal is to develop prevascularized constructs for implantation [7], while other strategies focus on rapid vascular

*Corresponding author: Daniel J. Gould, Baylor College of Medicine, Medical Scientist Training Program, One Baylor Plaza, Houston Texas 77030, USA, Tel: 916-806-9649; E-mail: djgould@bcm.edu

Received February 13, 2012; Accepted February 13, 2012; Published February 20, 2012

Citation: Gould DJ (2012) Congenital Facial Paralysis. Anaplastology 1:e102. doi: 10.4172/2161-1173.1000e102

Copyright: () 2012 Gould DJ. This is an open-access article distributed under the terms of the Creative Commons Attribution License, which permits unrestricted use, distribution, and reproduction in any medium, provided the original author and source are credited. 
invasion and perfusion [8]. The most promising research has combined these techniques to develop of rapidly vascularized scaffolds that recreate vascular space-filling properties similar to those found in normal, functional tissues, to provide adequate perfusion of implanted constructs [9-11].

Previous attempts have been made to provide prevascularized tissue engineering scaffolds. These attempts have included the use of mesenchymal stem cells in conjunction with Human Umbilical Vein derived Endothelial Cells (HUVECs- a common source of endothelial cells) to form vessel beds in the cranial window of skid mice [12]. Also similar attempts have been made with the use of human embryonic stem cells which were differentiated into endothelial cells, then implanted into the skid mouse through a cranial window and likewise showed vascular integration [13]. Although both of these methods were successful at demonstrating vessel integration in the brain, neither used brain derived endothelial cells, and both created functional vessels in this system, but the monitoring of the mice using a cranial window is complex and requires many precautions and considerations, as the mice have compromised immune systems.

One new model allows for facile monitoring in non-immune compromised mice to determine if vascular networks for tissue engineering applications can stand the test of implantation. This model involves the in vivo study of vascularization in non-immune compromised, fluorescently labeled mice via a corneal micropocket assay [14]. With proper mastery, this technique allows for appropriate visualization of vessels in an ideal implanted environment for anastamosis and integration. Demonstrates the technique principles and through practice, skilled technicians can complete the surgery in five minutes allowing for a high throughput approach. Furthermore, with automated image analysis tools and good sample sizes, this technique can allow for detailed analysis of the microvasculature [15].

Though the technology is rapidly evolving in order to address the need for tissue engineering, current clinical approaches are limited by expertise and costs. As the revelations from the bench side filter into clinical procedures and technologies, applications in humans will continue to develop. Importantly technology has to be developed which includes instruments, devices and bioreactors, along with proprietary formulations and biochemically modified scaffolds in order for the prospect of a tissue engineering industry to be sustainable and profitable. Without patented technology and tissue engineering scaffolds, the basic techniques and manipulations hold little promise for development in a life sciences technology approach. This being said, the horizon is vast for tissue engineering and the promise of tissue repair and organ replacement is particularly attractive given the increasing portion of our aging and diseased population in the US and throughout the world.

\section{References}

1. Graham W (2010) Organ Facts. United Network of Organ Sharing.

2. Langer R, Vacanti JP (1993) Tissue Engineering. Science 260: 920-926.

3. Mikos AG, Herring SW, Ochareon P, Elisseeff J, Lu HH, (2006) Engineering Complex Tissues. Tissue Eng 12: 3307-3339.

4. Langer R, Vacanti JP (1993) Tissue engineering. Science 260: 920-926.

5. Vacanti J, Vacanti CA, Robert L, Robert L, Joseph V (2007) Principles of Tissue Engineering (3rdedn), Academic Press, Burlington.

6. Vacanti $J(2010)$ Tissue engineering and regenerative medicine: from first principles to state of the art. J Pediatr Surg 45: 291-294.

7. Hoganson DM, Pryor HI 2nd, Vacanti JP (2008) Tissue engineering and organ structure: a vascularized approach to liver and lung. Pediatr Res 63: 520-526.

8. Atala A, Bauer SB, Soker S, Yoo JJ, Retik AB (2006) Tissue-engineered autologous bladders for patients needing cystoplasty. Lancet 367: 1241-1246.

9. Saik JE, Gould DJ, Keswani AH, Dickinson ME, West JL (2011) Biomimetic Hydrogels with Immobilized EphrinA1 for Therapeutic Angiogenesis. Biomacromolecules 12: 2715-2722.

10. Leslie-Barbick JE, Saik JE, Gould DJ, Dickinson ME, West JL (2011) The promotion of microvasculature formation in poly(ethylene glycol) diacrylate hydrogels by an immobilized VEGF-mimetic peptide. Biomaterials 32: 57825789 .

11. Saik JE, Gould DJ, Watkins EM, Dickinson ME, West JL (2011) Covalently immobilized platelet-derived growth factor-BB promotes angiogenesis in biomimetic poly(ethylene glycol) hydrogels. Acta Biomater 7: 133-143

12. Au P, Tam J, Fukumura D, Jain R (2008) Bone marrow-derived mesenchymal stem cells facilitate engineering of long-lasting functional vasculature. Blood 111: $4551-4558$

13. Wang ZZ, Au P, Chen T, Shao Y, Daheron LM, et al. (2007) Endothelial cells derived from human embryonic stem cells form durable blood vessels in vivo. Nat Biotechnol 25: 317-318.

14. Poché RA, Saik JE, West JL, Dickinson ME (2010) The Mouse Cornea as a Transplantation Site for Live Imaging of Engineered Tissue Constructs. Cold Spring Harb Protoc 4: 5416.

15. Gould DJ, Vadakkan TJ, Poché RA, Dickinson ME (2010) Multifractal and lacunarity analysis of microvascular morphology and remodeling. Microcirculation 18: 136-151. 